\title{
AN ALTERNATE CHARACTERIZATION OF THE CANTOR SET
}

\author{
ALAN H. SCHOENFELD AND GARY GRUENHAGE
}

ABSTRACT. Let $X$ be a compact metric space such that, up to homeomorphism, $X$ has only two nonempty open subsets. Then $X$ is homeomorphic to the Cantor discontinuum.

It is well known that any compact, perfect, totally disconnected metric space is homeomorphic to the Cantor "middle thirds" set $K$. The Cantor set is also known to have the following property: Up to homeomorph ism, $K$ has only two nonempty open subsets [1]. We will show that, among compact metric spaces, this property characterizes $K$.

Definition. A metric space $X$ has property $W$ if:

(a) $X$ has at least one nonempty compact open subset and at least one noncompact open subset (one of the se may be $X$ ).

(b) Any two nonempty compact open subsets of $X$ are homeomorphic.

(c) Any two noncompact open subsets of $X$ are homeomorphic.

Theorem. Let $X$ be a compact metric space. Then $X$ is homeomorphic to $K$ if and only if $X$ has property $W$.

Proof. The property is preserved by homeomorphism, and is thus necessary. Suppose now that $X$ has property $W$. Any isolated point of $X$ would, by (b), be homeomorphic to $X$, making $X$ a one-point space. This would contradict (a), so $X$ is perfect. We now show that $X$ is disconnected.

Let $x$ and $y$ be distinct points of $X, d$ the distance from $x$ to $y, U_{x}$ and $U_{y}$ the open balls of radius $d / 3$ about $x$ and $y$ respectively, and $U=$ $U_{x} \cup U_{y}$. If $U$ is homeomorphic to $X$, then $X$ is disconnected. If $U$ is not homeomorphic to $X$, then $U$ is noncompact. Since $X$ is perfect, we have for each $x \in X$ that $X \backslash\{x\}$ is noncompact and open, therefore (being homeomorphic to $U$ ) disconnected. Thus every point of $X$ is a cut-point of $X$. Here too $X$ must be disconnected, as every metric continuum has at least two non-cut-points.

Finally, take a point $x \in X$ and consider the quasicomponent (equals the component) of $x$, say $C$. The set $C$ cannot be open, for then it would be homeomorphic to $X$ and disconnected. Thus $X \backslash C$ is not compact and is

Received by the editors April 9, 1974 and, in revised form, September 25, 1974. $54 \mathrm{~F} 50$

AMS (MOS) subject classifications (1970). Primary 54G05, 54A10; Secondary

Key words and phrases. Cantor set. 
homeomorphic to $X \backslash\{x\}$. Now each $y \in X \backslash C$ has a compact ne ighborhood $V \subset X \backslash C$ (the complement of a closed-and-open $U \subset X$ such that $x \in U$ and $y \notin U)$. The same holds for $X \backslash\{x\}$, which shows that $X$ is totally disconnected. As a compact, perfect, totally disconnected metric space, $X$ is homeomorphic to $K$.

Corollary. Let $X$ be a noncompact metric space. Then $X$ is homeomorphic to $K \backslash\{0\}$ if and only if $X$ has property $W$.

The proof is trivial.

\section{REFERENCE}

1. Stephen Willard, General topology, Addison-Wesley, Reading, Mass., 1970, p. 219. MR $41 \# 9173$.

DEPARTMENT OF MATHEMATICS, UNIVERSITY OF CALIFORNIA, DAVIS, CALIFORNIA 95616

Current address: Group in Science and Mathematics Education, c/o Physics Department, University of California, Berkeley, California 94720 